\title{
Phytochemical compositions of extract from peel of hawthorn fruit, and its antioxidant capacity, cell growth inhibition, and acetylcholinesterase inhibitory activity
}

Panpan $\mathrm{Wu}^{1,2}$, Fajie $\mathrm{Li}^{3,4}$, Jianyong Zhang ${ }^{5}$, Bin Yang ${ }^{2 *}$, Zhaojie $\mathrm{Ji}^{1}$ and Weidong Chen ${ }^{1}$

\begin{abstract}
Background: Hawthorn fruit (HF) is a well-known traditional medicine in China with the effects of improving digestion and regulating qi-flowing for removing blood stasis. Modern pharmacological experiments showed that HF extract has various pharmaceutical properties and flavonoids are considered as the main bioactive compounds. In this paper, Diaion HP-20 adsorption chromatography was used to enrich flavonoids in PHF, and the phytochemical composition of EPHF was analyzed by high performance liquid chromatography (HPLC) and liquid chromatography tandem mass spectrometry (LC-MS). In addition, EPHF's antioxidant capacity, acetylcholinesterase (AChE) inhibitory activity and cytotoxic activity were evaluated.
\end{abstract}

Methods: EPHF was obtained by Diaion HP-20 adsorption chromatography. Phytochemical composition of EPHF was analyzed qualitatively and quantitatively using HPLC and LC-MS. Radical scavenging capacity of EPHF was estimated using 2,2-diphenyl-1-picryhydrazyl (DPPH) assay and oxygen radical absorbance capacity (ORAC) assay. The AChE inhibitory activity of EPHF was evaluated by Ellman method. Cytotoxic activity of EPHF was assessed by means of MTT assay.

Results: Eight kinds of components were identified, in which ideain with the value of $179.4 \mathrm{mg} / \mathrm{g}$ was identified to be present in the highest level in EPHF, followed by (-)-epicatechin, chlorogenic acid, cyanidin 3-arabinoside, hyperoside and isoquercitrin at the concentrations of 40.9, 10.0, 1.4, 0.4 and $0.2 \mathrm{mg} / \mathrm{g}$, respectively. The contents of these compounds in EPHF were much higher than those in PHF and HF. In addition, EPHF exhibited strong antioxidant and AChE inhibitory activity (ORAC value: $11.65 \pm 2.37 \mu \mathrm{M}$ Trolox equivalents $(\mathrm{TE}) / \mathrm{mg}$, DPPH IC $\mathrm{C}_{50}$ value: $6.72 \mu \mathrm{g} / \mathrm{mL}$, anti-AChE activity IC 50 value: $11.72 \mu \mathrm{g} / \mathrm{mL}$ ) compared with PHF and HF. Moreover, EPHF exhibited high levels of cytotoxicity on MCF-7 and SKOV-3 human tumour cell lines in a dose-dependent manner with the $\mathrm{IC}_{50}$ of 2.76 and $80.11 \mu \mathrm{g} / \mathrm{mL}$, respectively.

Conclusions: Macroporous resin is useful for the extraction and separation of the total flavonoids from PHF. The contents of flavonoids especially anthocyanin in EPHF were increased significantly compared with the PHF, and EPHF exhibited strong antioxidant, AChE inhibitory activity and cytotoxicity on human tumour cells.

Keywords: Hawthorn peel, Macroporous resin extract, Phytochemical composition, Antioxidant capacity, Cell growth inhibition, AChE inhibitory activity

\footnotetext{
* Correspondence: ybinmm@126.com

${ }^{2}$ Institute of Chinese Materia Medica, China Academy of Chinese Medical

Sciences, No. 16 Nanxiaojie, Dongzhimennei Ave., Dongcheng District,

Beijing 100700, China

Full list of author information is available at the end of the article
} 


\section{Background}

Medicinal plants are one of the most important sources of medicinal agents and their beneficial healing effects have been well demonstrated in ancient human civilizations worldwide. Hawthorn is distributed in the Northern Hemisphere mostly in China, Europe and North America [1] and has a long history of usage as a traditional medicine. In Europe and America, the extract of hawthorn leaves and flowers (eg. WS 1442, LI 132) are used for the treatment of hypertension and heart disease [2-4]. In China, hawthorn fruit (HF) has the effects of improving digestion and regulating qi-flowing for removing blood stasis. HF not only commonly be used as a traditional digestant, but also be combined with other Chinese medicines to treat cardiovascular diseases, for example, HF and Danshen (the root of Salvia miltiorrhiza Bge.) are used together to treat coronary disease and hyperlipemia. Modern pharmacological experiments showed that HF extract has anti-tumour, antioxidant [5], anti-aging and prevention of Alzheimer's effects [6-8]. HF extract also has the beneficial effects on the cardiovascular system [9-12].

Flavonoids are considered as the main bioactive compounds of HF, however, the content of flavonoid in HF is rather low (only about $10 \mathrm{mg} / \mathrm{g}$ ) [13, 14], while the content of sugar is high, which impedes the clinic usage of HF further. If we can get a flavonoid-rich extract from HF, we will make effective utilization of HF. So, the purpose of our research is to get a flavonoid-rich extract from $\mathrm{HF}$, to estimate its pharmacological activities, to find its potential possibility on clinical application. Our previous work showed that the flavonoids found in HF could also be found in the peel of hawthorn fruit (PHF) with higher concentration. In addition, macroporous resin has successfully been applied in the preparative separation of total flavonoids in natural products [15]. In the present study, extract with high content of flavonoids, especially anthocyanin, from the PHF (EPHF) was obtained by Diaion HP-20 adsorption chromatography with $20 \%$ aqueous ethanol elution. Phytochemical composition of EPHF was analyzed qualitatively and quantitatively using high performance liquid chromatography (HPLC) and liquid chromatography tandem mass spectrometry (LC-MS). At the same time, the antioxidant capacity, acetylcholinesterase (AChE) inhibitory activity and cytotoxic activity of EPHF were evaluated. To the best of our knowledge, this is the first time to get the extract with rich content of anthocyanin from HF and evaluate its pharmacological activity.

\section{Methods}

Materials and reagents

Fresh fruit of Crataegus pinnatifida Bge. var. major N. E. Br. was collected from Beijing China, and identified by Prof. Bin Yang. A voucher specimen (NO. 20160118) was deposited at Institute of Chinese Materia Medica, China Academy of Chinese Medical Sciences. The MCF7, SKOV-3 cell lines were obtained from the State Key Laboratory of Biotherapy in Sichuan University (Sichuan, China). Cyanidin 3-arabinoside and ideain were purchased from Sigma (St. Louis, MO, USA). Chlorogenic acid, (-)-epicatechin, hyperoside and isoquercitrin were purchased from National Institute for Food and Drug Control (Beijing, China). Procyanidin B2 was purchased from Phytolab (Vestenbergsgreuth, Germany). Diaion HP20 was purchased from Mitsubishi Chemical Co. Ltd. (Tokyo, Japan). Fluorescein disodium salt (FL), 2,2'-Azobis(2-methylpropionamidine) dihydrochloride (AAPH), 3(4,5-dimethyl-2-thiazolyl)-2,5-diphenyl-2-H-tetrazolium bromide (MTT), 2,2-Diphenyl- 1-picrylhydrazyl (DPPH), AChE, acetyhhiocholine (ATCI), 6-hydroxy-2,5,7,8tetramethylchroman-2-carboxylic acid (Trolox), 5,5'dithiobis- (2-nitrobenzoic acid) (DTNB) were purchased from Sigma (St. Louis, MO, USA). Dulbecco's modified Eagle's medium (DMEM), trypsin, penicillin and streptomycin were purchased from HyClone Laboratories Inc., (South Logan, UT, USA). Tris was purchased from Amresco (Solon, OH, USA). HPLC grade acetonitrile and methanol were purchased from Fisher (Fisher Scientific USA). All the other chemicals used from commercial sources were of analytical or higher grade.

\section{Sample preparation \\ Preparation of EPHF}

The peel and flesh of HF were separated and then the peel was lyophilized by lyophilizer (Christ, Germany). The dried PHF was milled into fine powder in a highspeed disintegrator and passed through a 40-mesh sieve before extraction. A sample of $1.0 \mathrm{~g}$ of powder was extracted three times with $10 \mathrm{~mL}$ of $95 \%$ ethanol containing $0.1 \%(\mathrm{v} / \mathrm{v})$ trifluoroacetic acid at room temperature for each extraction. The whole process of extraction needs to be protected from light. The extract solutions obtained from three extractions were combined, and filtered. Subsequently, the extract solution was concentrated with a vacuum rotary evaporator (Buchi, Swit Zerland) at $40{ }^{\circ} \mathrm{C}$, and then chromatographed on a Diaion HP-20 macroporous resin column, eluted subsequently with water, $20 \%$, and $60 \%$ ethanol all containing $0.1 \%(\mathrm{v} / \mathrm{v})$ trifluoroacetic acid. The fraction eluted by $20 \%$ ethanol containing $0.1 \%(\mathrm{v} / \mathrm{v})$ trifluoroacetic acid was collected, concentrated with a vacuum rotary evaporator and lyophilized.

\section{Preparation of the tested sample}

The accurately weighed EPHF was dissolved in methanol and filtered by $0.22 \mu \mathrm{m}$ membrane. 
The accurately weighed PHF powder (ca. $1.0 \mathrm{~g}$ ) was sonicated with $20 \mathrm{~mL}$ of $60 \%$ ethanol $(\mathrm{pH} 2)$ for $10 \mathrm{~min}$ at $30{ }^{\circ} \mathrm{C}$, and then centrifuged $10 \mathrm{~min}$ at $10,000 \mathrm{rpm}$, and filtered by $0.22 \mu \mathrm{m}$ membrane.

\section{Preparation of the standard solution}

The standards of cyanidin 3-arabinoside, ideain, chlorogenic acid, (-)-epicatechin, hyperoside , isoquercitrin and procyanidin B2 were accurately weighed (ca. $10 \mathrm{mg}$ ), subsequently, dissolved and diluted with methanol.

\section{Identification of compounds by LC-MS}

The analysis was performed on an ultimate 3000 ultraperformance liquid chromatography system coupled to an LTQ Orbitrap mass spectrometer via electrospray ionization (ESI) interface from Thermo Fisher Scientific (Bremen, Germany). This chromatography system consisted of an autosampler, a PDA detector, a column compartment and a quaternary pump. The mass spectra were recorded in simultaneous positive ionization fullscan mode throughout the $m / z$ range $50 \sim 800$, ion spray voltage $3.5 \mathrm{kV}$, capillary temperature $350{ }^{\circ} \mathrm{C}$, sheath gas flow rate $50 \mathrm{psi}$, aux gas flow rate $10 \mathrm{psi}$.

\section{Determination of compounds by HPLC}

The analysis was performed on an HPLC system (Shimadzu LC-20AT, Tokyo, Japan) consisting of a pump (LC-20A, Tokyo, Japan), a photodiode array detector (SPD-M20A photodiode array detector, Tokyo, Japan), and an Agilent Zorbax Extend C18 column (4.6 $\times$ $250 \mathrm{~mm}, 5 \mu \mathrm{m}$ ) with a solvent flow rate of $0.8 \mathrm{~mL} / \mathrm{min}$ at $35{ }^{\circ} \mathrm{C}$. Water and acetonitrile both with $2 \%$ formic acid were used as the mobile phase $A$ and $B$, respectively, and the gradient elution program was $5-8 \%$ B for 0-5 min, $8-11 \%$ B for $5-30 \mathrm{~min}, 11 \%-18 \% \mathrm{~B}$ for $30-$ $45 \mathrm{~min}$. Ideain and cyanidin 3-arabinoside were detected at the wavelength of $520 \mathrm{~nm}$, hyperoside and isoquercitrin were detected at the wavelength of $360 \mathrm{~nm},(-)$-epicatechin was detected at the wavelength of $280 \mathrm{~nm}$, and chlorogenic acid was detected at the wavelength of $330 \mathrm{~nm}$. Data signals were acquired and processed using LC-solution software.

\section{Pharmacological activity of EPHF Determination of antioxidant capacity}

The antioxidant capacities of samples were evaluated by measuring DPPH, AAPH free radical scavenging activity. These two assays were carried out according to our previously reported methods [16, 17]. Trolox was used as positive control in both of the two antioxidant experiments, and the capacity of scavenging AAPH was expressed as Trolox equivalents ( $\mu$ mol Trolox/mg extract).

\section{Determination of cell growth inhibition activity}

MCF-7 and SKOV-3 cells were cultured in DMEM supplemented with $10 \%$ heat inactivated $\mathrm{FBS}$, at $37{ }^{\circ} \mathrm{C}$ in a humidified atmosphere of $95 \%$ air and $5 \% \mathrm{CO}_{2}$. The cells were seeded onto 96-well culture plate at a density of $3 \times 10^{3}$ cells/well and allowed to adhere for $24 \mathrm{~h}$. The cells were incubated with different doses $(0.5,5,12.5,25$ and $50 \mathrm{mg} / \mathrm{L}$ ) of samples for $48 \mathrm{~h}$. All the reactions were performed in six replicates.

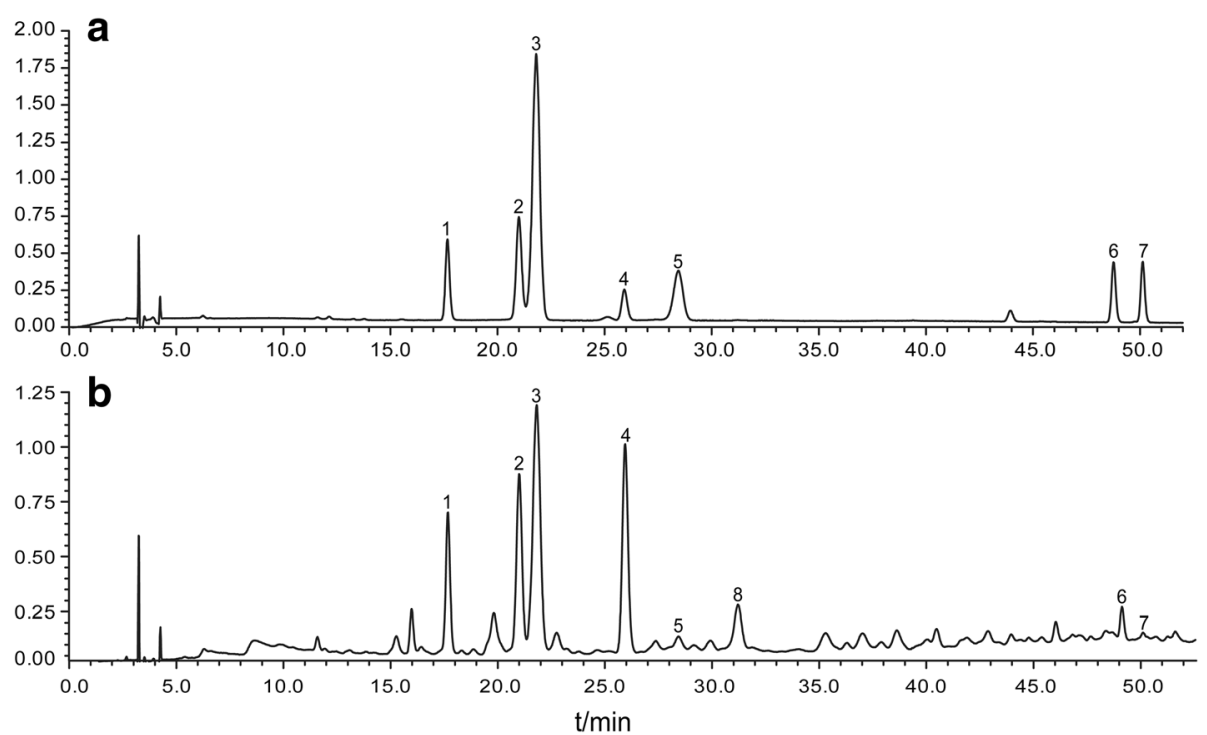

Fig. 1 The HPLC chromatograms of reference (a) and sample solution (b) at $280 \mathrm{~nm}$. Peaks 1 8: (1) chlorogenic acid; (2) procyanidin B2; (3) ideain; (4) (-)-epicatechin; (5) cyanidin 3-arabinoside; (6) hyperoside; (7) isoquercitrin; (8) procyanidin C1 
Table 1 Calibration curves, LODs and LOQs of the standard compounds

\begin{tabular}{|c|c|c|c|c|c|}
\hline Compound & Calibration curve & $r$ & Linear range/mg & LOQ/ng & $\mathrm{LOD} / \mathrm{ng}$ \\
\hline Ideain & $Y=5000000 x-5992.1$ & 0.9999 & $0.0049 \sim 0.63$ & 4.93 & 2.47 \\
\hline Cyanidin 3-arabinoside & $Y=5000000 x-12627$ & 0.9999 & $0.0049 \sim 0.63$ & 4.89 & 2.44 \\
\hline Hyperoside & $Y=3000000 x-70021$ & 0.9998 & $0.0049 \sim 5.02$ & 4.90 & 2.45 \\
\hline Isoquercitrin & $Y=3000000 x-68963$ & 0.9997 & $0.0049 \sim 5.01$ & 4.89 & 2.45 \\
\hline (-)-Epicatechin & $Y=835115 x-7850.7$ & 0.9999 & $0.0099 \sim 5.12$ & 9.99 & 2.50 \\
\hline Chlorogenic acid & $Y=4000000 x-131307.7$ & 0.9995 & $0.0050 \sim 2.57$ & 5.01 & 2.50 \\
\hline
\end{tabular}

The cell growth inhibition effect of EPHF was evaluated by MTT reduction. After $48 \mathrm{~h}$ incubation, MTT solution in PBS was added in a final concentration of $0.5 \mathrm{mg} / \mathrm{mL}$. The plate was incubated at $37{ }^{\circ} \mathrm{C}$ for $4 \mathrm{~h}$, followed by the addition of $150 \mu \mathrm{L}$ DMSO to dissolve the dark blue formazan crystals. The absorbance at $570 \mathrm{~nm}$ was measured on a Varioskan Flash Multimode Reader (Thermo Scientific, Finland). Cell viability was calculated as the percentage of untreated control.

\section{Determination of AChE inhibitory activity}

AChE-inhibiting activities were measured by the slightly modified spectrophotometric method developed by Ellman et al. [18, 19]. Briefly, $10 \mu \mathrm{L}$ of Tris/HCl buffer containing $20 \mathrm{mM}$ Tris $(\mathrm{pH}$ 7.5), $80 \mu \mathrm{L}$ of DTNB $(1 \mathrm{mM}), 4 \mu \mathrm{L}$ of sample, and $4 \mu \mathrm{L}$ of $\mathrm{AChE}(2000 \mathrm{U})$ were mixed in the wells and incubated for $10 \mathrm{~min}$ at room temperature. The reaction was initiated by addition of $2 \mu \mathrm{L}$ of ATCI $(20 \mathrm{mM})$. The microplate was then read at a wavelength of $412 \mathrm{~nm}$ every $30 \mathrm{~s}$ for 15 min by a Varioskan Flash Multimode Reader (Thermo Scientific, Finland). These samples were dissolved in Tris/ $\mathrm{HCl}$ buffer (containing $2 \% \mathrm{MeOH}, \mathrm{v} / \mathrm{v}$ ). All the reactions were performed in six replicates. Galanthamine was used instead of EPHF in positive control group.

\section{Results and discussion}

\section{The preparation of EPHF}

In our previous study, Diaion HP-20 macroporous resin was shown to be a good material to enrich flavonoids in HF [20]. In the present paper, the flavonoids in PHF were enriched using Diaion HP-20 macroporous resin. The sample was eluted with water, $20 \%$, and $60 \%$ ethanol all containing $0.1 \%(\mathrm{v} / \mathrm{v})$ trifluoroacetic acid subsequently, and the corresponding eluents were analyzed by HPLC. The results showed that flavonoids with diversified structures were enriched in 20\% ethanol eluent, while sugar was enriched in the fraction eluted by water, and little flavonoids were detected in $60 \%$ ethanol eluent. Therefore, the fraction eluted by $20 \%$ ethanol was selected for the next experiment.

\section{Qualitative and quantitative analysis of EPHF Optimization of the sample preparation}

To get a high-extraction efficiency of the target compounds from the sample, different extraction solvents (hybrid solutions of ethanol and acid water), the solvent-to-sample ratio, extraction methods (ultrasonic, reflux, and infrared-assisted reflux extraction), temperature $\left(20,30,40\right.$ and $\left.50{ }^{\circ} \mathrm{C}\right)$ and time $(30,60$ and $90 \mathrm{~min}$ ) were studied. Sixty percent ethanol $(\mathrm{pH} 2)$ as the solvent and ultrasonic extraction for $10 \mathrm{~min}$ at $30{ }^{\circ} \mathrm{C}$ was found to be suitable for the extraction of the target compounds.

\section{Qualitative analysis of EPHF}

Chromatographic conditions were optimized for the qualitative analysis of EPHF. In this process, several mobile phases (hybrid systems of organic phase and acid water) were studied, and the best separation result was

Table 2 Precision and recovery for the developed HPLC method

\begin{tabular}{|c|c|c|c|c|c|}
\hline \multirow[t]{2}{*}{ Compound } & \multicolumn{2}{|c|}{ Precision RSD (\%) } & \multirow{2}{*}{$\begin{array}{l}\text { Reproducibility } \\
\text { RSD (\%) }\end{array}$} & \multicolumn{2}{|l|}{ Recovery } \\
\hline & Intra-day & Inter-day & & Average (\%) & RSD (\%) \\
\hline Ideain & 1.45 & 1.45 & 1.38 & 98.39 & 3.60 \\
\hline Cyanidin 3-arabinoside & 1.53 & 1.44 & 1.74 & 102.78 & 1.92 \\
\hline Hyperoside & 1.46 & 1.93 & 2.12 & 100.68 & 0.73 \\
\hline Isoquercitrin & 1.44 & 1.81 & 0.81 & 103.05 & 1.49 \\
\hline (-)-Epicatechin & 1.36 & 1.92 & 1.72 & 101.51 & 3.15 \\
\hline Chlorogenic acid & 1.76 & 1.72 & 0.34 & 99.05 & 0.99 \\
\hline
\end{tabular}


Table 3 Contents of compounds in samples/\% $(n=2)$

\begin{tabular}{llllllll}
\hline Samples & Ideain & Cyanidin 3-arabinoside & Hyperoside & Isoquercitrin & (-)-Epicatechin & Chlorogenic acid & Total \\
\hline EPHF & 17.94 & 0.14 & 0.04 & 0.02 & 4.09 & 1.00 & 23.23 \\
PHF & 0.087 & 0.010 & 0.038 & 0.022 & 0.64 & 0.084 & 0.881
\end{tabular}

EPHF extract from the peel of hawthorn fruit, PHF peel of hawthorn fruit

obtained when using an acetonitrile-formic acid solution as mobile phase at $35^{\circ} \mathrm{C}$ (Fig. 1).

Peaks $1 \sim 7$ were identified as chlorogenic acid, procyanidin B2, ideain, (-)-epicatechin, cyanidin 3arabinoside, hyperoside and isoquercitrin by comparing their retention times and UV spectra with those of standard compounds (Fig. 1). Peak 8 was identified as procyanidin $\mathrm{C} 1$ according to its precursor ion with an $m / z$ value of $867.21[\mathrm{M}+\mathrm{H}]{ }^{+}$and other characteristic ions $289.07,577.13,579.15,889.19$ [21, 22].

\section{Method validation for quantitative analysis}

Method validation was performed on parameters such as linearity, recovery and precision. Calibration curves of 6 standard compounds (ideain, (-)-epicatechin, chlorogenic acid, cyanidin 3-arabinoside, hyperoside and isoquercitrin) were constructed from peak areas versus compound amounts. The limit of quantification (LOQ) and limit of detection (LOD) for each compound were determined at signal-to-noise ratios of 10 and 3 , respectively. The calibration data were shown in Table 1. Relative standard deviation (RSD) value was calculated, and it was considered as the measure of precision. To assess the intra-day precision, the standard solution was injected seven times within a day. The inter-day precision test was assessed by testing over three consecutive days (Table 2). The assay method precision determination was carried out using six independent sample solutions. The accuracy of quantitation in terms of recovery was assessed. Standard solutions were spiked into the sample solution containing half the mass of the plant material with known amounts of the tested analytes, and the sample was extracted according to the procedure described in sample preparation. The amount of each analyte in the standard solution was almost equal to that in the sample solution. The RSD values of reproducibility were all less than $3 \%$. The average recovery rate of the six analytes ranged from 98.39 to $103.05 \%$, with RSD values varying between 0.73 and $3.60 \%$ (Table 2).

In this paper, the contents of procyanidin B2 and procyanidin $\mathrm{C} 1$ in sample were not determined, because of the limited availability of standard of procyanidin $\mathrm{C} 1$ and the poor chromatographic separation of procyanidin $\mathrm{B} 2$.

\section{Quantitative analysis of samples}

The contents of the 6 compounds in both of EPHF and PHF were determined, and result was shown in Table 3. The results indicated that the contents of anthocyanin and (-)-epicatechin and the total amount of the $6 \mathrm{com}$ pounds in EPHF were much higher than those in PHF. Obviously, the purification method of macroporous resin is useful for the extraction and separation of the flavonoids from PHF.

\section{Pharmacological activity of EPHF Antioxidant capacity of EPHF}

In this study, the antioxidant capacities of EPHF and PHF were evaluated by measuring DPPH free radical scavenging activity and oxygen radical absorbance capacity (ORAC). The results indicated that EPHF exhibited the strongest DPPH and AAPH free radical scavenging capacity, followed by PHF and HF in a decreasing order [23] (Table 4). Our previous studies have shown that (-)-epicatechin, chlorogenic acid, hyperoside, isoquercitrin and procyanidin B2 all have strong free radicalscavenging activity [16, 17], accordingly, the high concentrations of the antioxidant ingredients in EPHF contributed to the strong antioxidant activity of EPHF, i.e., the higher the concentrations of the antioxidant ingredients, the stronger the antioxidant activity of the sample.

The excessive free radicals in organs may bind to and destroy body cells, and are considered to be the main cause of some diseases, such as aging, cancer and cardiovascular disease [24, 25]. Our results suggest that EPHF may be the potential source of antioxidant agents

Table 4 Free radical scavenging activity of samples $(n=6)$

\begin{tabular}{lllll}
\hline & $H F$ & PHF & EPHF & Trolox \\
\hline DPPH $\left(I C_{50}, \mu \mathrm{g} / \mathrm{mL}\right)$ & $330[17]$ & 66.30 & 6.72 & $6.72^{\mathrm{a}} / 8.69^{\mathrm{b}}$ \\
ORAC $(\mu \mathrm{mol} / \mathrm{mg})$ & $0.375 \pm 0.4[23]$ & $1.27 \pm 0.44$ & $11.65 \pm 2.37$ & \\
\hline
\end{tabular}

EPHF extract from the peel of hawthorn fruit, $P H F$ peel of hawthorn fruit, $H F$ hawthorn fruit

${ }^{\mathrm{a}} \mathrm{I} \mathrm{C}_{50}$ of Trolox in reference [17]

${ }^{\mathrm{b}} \mathrm{IC}_{50}$ of Trolox tested in the present experiment 
Table 5 AChE inhibitory activity of samples $(n=6)$

\begin{tabular}{lllll}
\hline & HF & PHF & EPHF & Galanthamine \\
\hline Anti-AChE $\left(I C_{50}, \mu \mathrm{g} / \mathrm{mL}\right)$ & 1385 & 616.40 & 11.72 & 0.29 \\
\hline
\end{tabular}

EPHF extract from the peel of hawthorn fruit, PHF peel of hawthorn fruit, HF hawthorn fruit

to be used as an alternative for the treatment of free radical-associated disease.

\section{AChE inhibitory activity of EPHF}

In this study, the AChE inhibitory activity of EPHF, PHF and HF were evaluated, and the result showed that EPHF exhibited the highest AChE inhibitory activity, followed in decreasing order by PHF and HF (Table 5).

AChE plays an important role in the growth and matureness of cell, and it also promotes the development of neurons and the regeneration of nerves. AChE inhibitor has been used as a drug for the symptomatic treatment of Alzheimer's disease [26]. Our results suggest that EPHF has a relatively strong anti-AChE effect, indicating its potential to be used as an alternative for the treatment of Alzheimer and related disorders.

\section{Effect of EPHF on cell growth of human carcinoma}

EPHF significantly inhibited MCF-7, SKOV-3 cells proliferation in a concentration-dependent manner with the $\mathrm{IC}_{50}$ value of 2.76 and $80.11 \mu \mathrm{g} / \mathrm{mL}$, respectively. In Li's study, the $\mathrm{IC}_{50}$ value of PHF and hawthorn fruit fleshes on MCF-7 cells were 88.6 and $175.5 \mu \mathrm{g} / \mathrm{mL}$, respectively [27], which indicated that EPHF might exhibit the stronger inhibitory effect against MCF-7 cells compared with PHF.

In summary, the pharmacological result suggests the potential of EPHF to be an alternative for the treatment of free radical-associated diseases, and Alzheimer and related disorders.

\section{Conclusions}

Macroporous resin is useful for the extraction and separation of the total flavonoids from PHF. The contents of flavonoids especially anthocyanin in EPHF were increased significantly compared with those in PHF and HF. More importantly, the pharmacological experiments showed that EPHF exhibited stronger DPPH and AAPH free radical scavenging activity and AChE inhibitory activity comparing with PHF and HF. Moreover, EPHF inhibited the proliferation of cancer cells. As the first report on the phytochemical compositions and pharmacological activity analysis of EPHF, the results of this study was laying the foundation for further research on the health benefits of hawthorn and its exploitation and utilization.
Abbreviations

AAPH: 2,2'-Azobis(2-methylpropionamidine) dihydrochloride; AChE: Acetylcholinesterase; ATCl: Acetyhhiocholine; DMEM: Dulbecco's modified Eagle's medium; DPPH: 2,2-diphenyl-1-picryhydrazyl; DTNB: 5,5'dithiobis-(2-nitrobenzoic acid); EPHF: Extract from the peel of hawthorn fruit; FL: Fluorescein disodium salt; HF: Hawthorn fruit; $\mathrm{IC}_{50}$ : Half-maximal inhibitory concentration; MTT: 3-(4,5-dimethyl-2-thiazolyl)-2,5-diphenyl-2-H-tetrazolium bromide; ORAC: Oxygen radical absorbance capacity; PHF: Peel of hawthorn fruit

Acknowledgements

The authors are grateful to the State Key Laboratory of Biotherapy in Sichuan University for providing the MCF-7, SKOV-3 cell lines.

Funding

This work was supported by an "863" Project of China (No. 2014AA022201).

\section{Availability of data and materials}

All data generated or analyzed during this study are included in this article, the materials and data of our study are available to other researchers upon request.

Authors' contributions

BY and PPW conceived and designed the experiments. Phytochemical composition of EPHF was analyzed by PPW. JYZ and FJL involved in the anticancer and anti-AChE activity experiment, ZJJ and WDC were participated in the experiment of antioxidant. The paper was written by PPW and reviewed by BY. All authors reviewed and approved the final version.

Competing interests

The authors declare that they have no competing interests.

Consent for publication

This information is not relevant.

Ethics approval and consent to participate

This information is not relevant.

\section{Publisher's Note}

Springer Nature remains neutral with regard to jurisdictional claims in published maps and institutional affiliations.

\section{Author details}

${ }^{1}$ Anhui University of Chinese Medicine, Hefei 230012, China. ${ }^{2}$ Institute of Chinese Materia Medica, China Academy of Chinese Medical Sciences, No. 16 Nanxiaojie, Dongzhimennei Ave., Dongcheng District, Beijing 100700, China. ${ }^{3}$ State Key Laboratory of Dao-di Herbs, National Resource Center for Chinese Materia Medica, China Academy of Chinese Medical Sciences, Beijing 100700, China. ${ }^{4}$ Flow Station of Post-Doctoral Scientific Research, China Academy of Chinese Medical Sciences, Beijing 100700, China. ${ }^{5}$ Pharmacy School, Zunyi Medical University, Guizhou 563000, China.

Received: 7 December 2016 Accepted: 7 March 2017

Published online: 11 March 2017

\section{References}

1. Liu PZ, Yang B, Kallio H. Characterization of phenolic compounds in Chinese hawthorn (Crataegus pinnatifida Bge. var. major) fruit by high performance liquid chromatography-electrospray ionization mass spectrometry. Food Chem. 2010;121(4):1188-97.

2. Bubik MF, Willer EA, Bihari $P$, Jurgenliemk G, Ammer $H$, Krombach F, Zahler S, Vollmar AM, Furst R. A novel approach to prevent endothelial hyperpermeability: The crataegus extract WS ${ }^{\oplus} 1442$ targets the camp/rap1 pathway. J Mol Cell Cardiol. 2012;52(1):196-205. 
3. Dahmer S, Scott E. Health effects of hawthorn. Am Fam Physician. 2010; 81(4):465-8.

4. Rigelsky JM, Sweet BV. Hawthorn: pharmacology and therapeutic uses. Am J Health Syst Pharm. 2002;59(5):417-22.

5. Liu J, Popovich DG, Jing H. Hawthorn flavonoid extract: antioxidant activity and growth inhibition effect on cancer cells. Food Sci. 2010;31(3):220-3.

6. Huang YS, Xie MY, Nie SP, Chang LI. Anti-oxidative activity of extracts from hawthorn. Food Sci Biotechnol. 2010;29(2):189-92.

7. $\mathrm{Ni} X Q$, Huo YR, Lu GQ. Advances in health funcations anthocyanin. J Anhui Agri Sci. 2010;38(35):20025-8.

8. Zhang ZS, Zuo YB, Wang H, Chen YY, Li WB. Anti-aging effect of hawthorn alcohol extract on drosophila melanogaster. Acta Nutrimenta Sinica. 2011; 33(4):397-9.

9. Wang $W$, Yang B, Wang L, Liang RX, Chen CY, Hu N, Cheng L, Yang Y, Yin XJ, Gao SR. Cooperative effect of salvia miltiorrhiza and hawthorn for resisting atherosclerosis in rats. Chin J Exp Tradit Med Formul. 2011;36(6):784-9.

10. Wu SJ, Li QJ, Xiao XF, Li M, Yang XR, Lv T. The chemical components and pharmacological action of hawthorn. Drug Eval Res. 2010;33(4):316-9.

11. Gao Y, Xiao Y. Effect of hawthorn and hawthorn flavonoids extract on rats with hyperlipidemia. Chin J Food Hyg. 2002;14(3):14-6.

12. Zhang JY, Yan RY, Wang L, Wang JL, Wang WD, Rui H, Liu HL, Yang HJ, Liang RX, Yang B. Experimental study on anti-atherosclerotic effect of compatibility of active components of Danshen and Shanzha. Chin J Chin Mater Med. 2013;38(12):1987-91.

13. Chen CY, Yang B, Zhou J. Study on quality evaluation of hawthorn. Chin J Exp Tradit Med Formul. 2009;15(12):1-4.

14. Li H. RP-HPLC analysis of flavonoids in hawthorn fruits. Chin J Pharma Anal. 2006;26(7):881-4

15. Fu B, Liu J, Li H, Li L, Lee FSC, Wang X. The application of macroporous resins in the separation of licorice flavonoids and glycyrrhizic acid. J Chromatogr A. 2005;1089(1-2):18-24.

16. Dai $\mathrm{HQ}$, Chen $\mathrm{CY}$, Yang B. AAPH scavenging activities of 22 flavonoids and phenolic acids and 9 extracts of Chinese materia medica. Chin J Chin Mater Med. 2010;35(17):2296-302.

17. Yuan YN, Chen CY, Yang B, Fumiyo K, Akira K. DPPH radical scavenging activities of 31 flavonoids and phenolic acids and 10 extracts of Chinese materia medica. Chin J Chin Mater Med. 2009;34(13):1695-700.

18. Ellman GL, Courtney KD, Jr VA, Featherstone RM. A new and rapid colorimetric determination of acetylcholinesterase activity. Biochem Pharmacol. 1961:7(2):88-95.

19. Liu Y, Zhou JL, Liu P, Sun S, Li P. Chemical markers' fishing and knockout for holistic activity and interaction evaluation of the components in herbal medicines. J Chromatogr A. 2010;1217(32):5239-45.

20. Wang JJ, Yan RY, Yang B. Anthocyanin from the fruit of Crataegus pinnatifida Bge. var. major N. E. Br. Lishizhen Med Materia Medica Res. 2015; 26(1):42-3.

21. Liu P, Kallio H, Yang B. Phenolic compounds in hawthorn (Crataegus grayana) fruits and leaves and changes during fruit ripening. J Agric Food Chem. 2011;59(20):11141-9.

22. Salminen JP, Karonen M, Lempa K, Liimatainen J, Sinkkonen J, Lukkarinen M, Pihlaja K. Characterisation of proanthocyanidin aglycones and glycosides from rose hips by high-performance liquid chromatography-mass spectrometry, and their rapid quantification together with vitamin C. J Chromatogr A. 2005;1077(2):170-80.

23. Chen CY, Li H, Yuan YN, Dai HQ, Yang B. Antioxidant activity and components of a traditional chinese medicine formula consisting of Crataegus pinnatifida and Salvia miltiorrhiza. BMC Complement Altern Med. 2013;13(7):99.

24. Alpay Z, Saed GM, Diamond MP. Female infertility and free radicals: potential role in adhesions and endometriosis. Reprod Sci. 2006;13(6):390.

25. Bhat R, Liong MT, Abdorreza MN, Karim AA. Evaluation of free radical scavenging activity and antioxidant potential of a few popular green leafy vegetables of malaysia. Int J Food Prop. 2013;16(6):1371-9.

26. Ingkaninan K, Temkitthawon P, Chuenchom K, Yuyaem T, Thongnoi W. Screening for acetylcholinesterase inhibitory activity in plants used in Thai traditional rejuvenating and neurotonic remedies. J Ethnopharmacol. 2003; 89(2-3):261-4

27. Li T, Zhu J, Guo L, Shi X, Liu YF, Yang XB. Differential effects of polyphenols-enriched extracts from hawthorn fruit peels and fleshes on cell cycle and apoptosis in human MCF-7 breast carcinoma cells. Food Chem. 2013;141(2):1008-18.

\section{Submit your next manuscript to BioMed Central and we will help you at every step:}

- We accept pre-submission inquiries

- Our selector tool helps you to find the most relevant journal

- We provide round the clock customer support

- Convenient online submission

- Thorough peer review

- Inclusion in PubMed and all major indexing services

- Maximum visibility for your research

Submit your manuscript at www.biomedcentral.com/submit
Biomed Central 